\title{
Managing Critical Nodes in UAV assisted Disaster Networks
}

\author{
Ali Hassan*, Rizwan Ahmad*, Waqas Ahmed ${ }^{\dagger}$, Maurizio Magarini ${ }^{\ddagger}$, and Muhammad Mahtab Alam ${ }^{\S}$ \\ *National University of Sciences and Technology (NUST), Islamabad, Pakistan, \\ ${ }^{\dagger}$ Pakistan Institute of Engineering and Applied Sciences, Islamabad, Pakistan, \\ ${ }^{\ddagger}$ Dipartimento di Elettronica, Informazione e Bioingegneria, Politecnico di Milano, Italy, \\ $\S$ Thomas Johann Seebeck Department of Electronics, Tallinn University of Technology, Tallinn 19086, Estonia, \\ Email: ahassan.msee18seecs@ seecs.edu.pk
}

\begin{abstract}
Device-to-device (D2D) communications serve as an alternative to cellular networks to enable communication for public safety networks (PSNs). A key requirement for PSNs is to offer alternative access to reach the responders if the communication infrastructure is partially or completely damaged due to a natural or man-made disaster. In this paper, we propose a novel unmanned aerial vehicle (UAV) assisted solution to ensure energy-efficient D2D connectivity in the disaster zone in the presence of critical nodes (CNs). The results show that the minimum average outage is achieved when the UAV is placed at the center of the region associated with the UAV, however, this scenario completely changes with the presence of CNs. Initially, with an increase in the number of CNs, the optimal UAV position shifts from the centre, however, increasing CNs causes the UAV to converge back to the center of the UAV associated region. To cater the service requirements of $\mathrm{CNs}$, we analyze the impact of increasing the mobile command center (MCC) coverage range and study its impact on UAV placement by varying the ratio of CNs and non-CNs. We found that the average outage probability decreases with the increase of the MCC range.
\end{abstract}

Index Terms-Public safety networks (PSNs), D2D, critical nodes, UAV, clustering.

\section{INTRODUCTION}

Over the past few years, every year approximately 60,000 people die from natural disasters such as earthquakes, torrential rains, floods landslides, and etc. This constitutes an average of $0.1 \%$ of the total deaths worldwide [1]. According to Statista [2], worldwide losses affected by man-made disasters amounted to approximately 7.73 billion U.S. dollars in 2019. In both the natural and man-made disasters, the communication infrastructure causes partial or complete failure. Both the partial and complete failure of telecommunications infrastructure leads to an unnecessary loss of lives, mainly due to the delays in post-disaster recovery. Due to the complete failure, there is no direct link between the on-scene available (OSA) devices and the responders, this makes the responders task difficult. In this scenario, it is difficult to predict the number of casualties, severely injured, and trapped persons. Similarly in the man-made disasters, the presence of terrorists is an active threat where there is no information about the number of terrorists, their location, and the weapons they are carrying.

978-1-7281-9444-8/20/\$31.00 @2020 IEEE
In the literature, several solutions try to address the problem of connectivity in disaster scenarios with the help of unmanned aerial vehicles (UAVs). The UAVs are used to provide connectivity to OSA devices from nearby functional base stations (BS) [3]-[7]. Another important point of concern for these types of networks is their network lifetime. The researchers have resorted on clustering to improve the network lifetime and energy consumption of such networks [7]-[15]. Authors in [16] provide a comparison of clustering strategies for the activation of energy-aware routing for PSNs in $\mathrm{D} 2 \mathrm{D}$ communication. The results show the benefits of the clustering schemes in the disaster situation in terms of power consumption, residual energy, and the number of dead nodes.

Similarly, a lot of research effort is focused on finding the best position of the UAV to provide extended connectivity and coverage. In [17] an algorithm is proposed based on client information and point of interest to find the best position of the UAV in a flying network. The aim of this paper to find the optimal position for UAVs where it facilitates the maximum number of clients determines the number of required UAVs to cover the whole scenario and also caters to the energy constraint of the UAV battery. In [18] authors propose to use a reinforcement learning algorithm to find the best position for the multiple Drone Small Cell which replace the actual BSs. The drone small cells can communicate with each other, however, the main decisions are taken at the central BS.

The OSA devices have limited batteries resources and due to frequent forwarding in D2D mode, some of the devices run out of the battery causing network reconfiguration. In D2D networks, some of the OSA devices have critical information or are severely energy critical. These nodes are referred to as critical nodes (CNs). To prevent network partition and frequent re-configurations it is important to intelligently allocate resources to $\mathrm{CNs}$.

This paper presents a practical solution for the abovementioned problems such as connectivity in a disaster zone, increasing the energy efficiency of the networks, and catering for information and energy CNs. The main contributions of the research are to:

- Determine the UAV position in the presence of $\mathrm{CNs}$ to minimize the average outage probability of the network. 
- To analyze the impact of varying mobile command center (MCC) coverage area and the percentage of non-CNs on the UAV placement.

- A weighted metric $\beta$ is defined that adjusts the UAV position in order to manage the trade-off between the outage of CNs and non-CNs. The relationship between the percentage of $\mathrm{CNs}$ and $\beta$ is evaluated in terms of outage probability.

Rest of the paper is organized as follows: Section II describes the generic system model for disasters including clustering, the average outage calculation, and UAV position. Section III, provides detailed analysis and simulation results. Finally, Section IV presents the conclusion and future directions.

\section{System ModeL}

Consider a disaster scenario as shown in Fig. 1, where the BS is not functional, and the victims are unable to communicate with the responders. We assume that the OSA devices are randomly deployed in the disaster area and these devices support multiple wireless interfaces such as LTE-D, LTE-A, Wi-Fi, Wi-Fi-direct, and BLE [19]. It is important to note that the OSA devices will have different capabilities and resources such as their information, battery level, and location. The OSA devices must be able to discover each other within their proximity. One of the possible solutions to develop temporary connectivity between responders and the victims through the D2D interface. A temporary BS in the form of MCC is deployed near the disaster scenario. Due to the constraint of receiver sensitivity, some of the OSA devices cannot directly communicate their information to the MCC. Therefore, it is possible for OSA devices to communicate their information in a multi-hop manner or with the aid of UAV.

The OSA devices use the proximity information to perform clustering. The clustering algorithm is deduced from the kmeans algorithm. The major difference from the k-mean algorithm lies in the selection of cluster head $(\mathrm{CH})$ and the selection of the number of clusters $\left(C_{k}\right)$. For the selection of $\left(C_{k}\right)$ an Elbow algorithm is used [20] The distance and energy information is used to elect the OSA devices as CHs or cluster members (CMs). The next important aspect is to determine the average outage probability for OSA devices communicating their information in a multi-hop manner to the MCC.

\section{A. Average Outage Probability}

The outage in each hop is determined by comparing the received signal power with the desired threshold $\left.\left(\gamma_{t h}\right)\right)$. The channel between $\mathrm{CM}$ to $\mathrm{CH}, \mathrm{CH}$ to $\mathrm{CH}, \mathrm{CH}$ to $\mathrm{MCC}$, and $\mathrm{CH}$ to UAV is Rayleigh fading, and the channel between UAV to MCC is Rician fading [21]. The noise between $\mathrm{CH}$ to $\mathrm{CH}, \mathrm{CH}$ to $\mathrm{CM}, \mathrm{CH}$ to $\mathrm{UAV} / \mathrm{MCC}$, and UAV to $\mathrm{MCC}$ is assumed to be AWGN. Assuming $S$ and $D$ indicate one of the communication links, then the received signal power is given as

$$
P_{r x, S-D}=\frac{P_{t x}\left|h_{S-D}\right|^{2}}{\sigma^{2} d_{S-D}^{n}}
$$

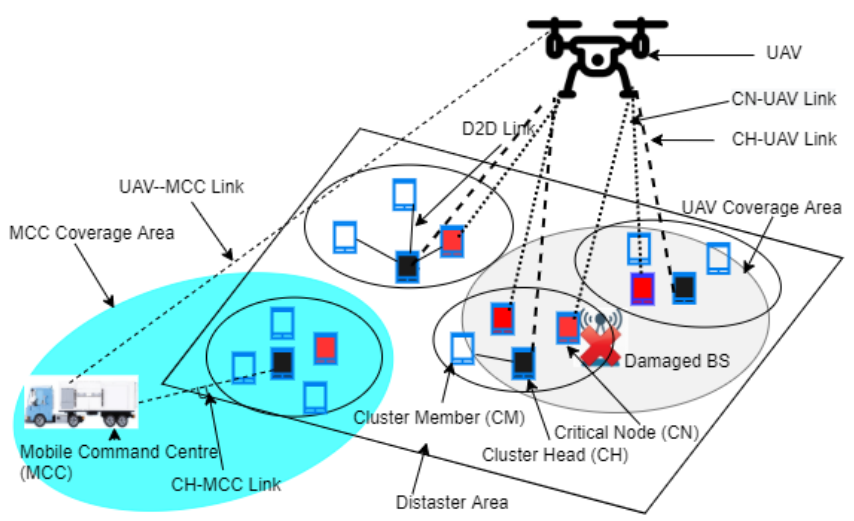

Fig. 1. Proposed Model Framework for disaster recovery communication using D2D clustering.

where, $P_{t x}$ is the device power and assumed constant for all OSA, $h_{S-D}$ is the Rayleigh or Rician fading coefficient, $d_{S-D}$ is the distance between source and destination, $n$ is the path loss exponent, and $\sigma^{2}$ is the variance of AWGN noise. In this paper, we are interested in an average end to end outage $\mathrm{E}\left(P_{o}\right)$ of $N$, where $\mathrm{E}($.$) is an expectation operator and P_{o}$ is the end to end outage probability. This term represents an average outage between an OSA device and the MCC. If $S$ and $D$ represents the source and destination in the $l^{\text {th }}$ hop, the outage probability is defined as

$$
\begin{gathered}
P_{o, l}=\operatorname{Pr}\left(P_{r x, S-D} \leq \gamma_{t h}\right) \\
P_{o, l}=\operatorname{Pr}\left(\frac{P_{t x}\left|h_{S-D}\right|^{2}}{\sigma^{2} d_{S-D}^{n}} \leq \gamma_{t h}\right) \\
P_{o, l}=\operatorname{Pr}\left(\left|h_{S-D}\right|^{2} \leq \frac{\gamma_{t h} \sigma^{2} d_{S-D}^{n}}{P_{t x}}\right)=1-e^{-\left(\frac{\gamma_{t h} \sigma^{2} d_{S-D}^{n}}{P_{t x}}\right)}
\end{gathered}
$$

For a general multi-hop scenario, end to end outage probability $P_{o}$ can be written as

$$
P_{o}=1-\prod_{l=1}^{\text {noofhops }} P_{o, l},
$$

where the last hop is between the UAV and MCC, the exponential term should be replaced by the Rician distribution cumulative distribution function. Since the OSA devices are randomly distributed in a disaster region, averaging over the distribution of randomly generated hops from an OSA device to MCC and corresponding hop distances are needed to determine the average end to end outage.

\section{B. CNs aware UAV positioning}

In this paper, we assume that the MCC is static and the disaster region is already clustered. The $\mathrm{CHs}$ are associated 


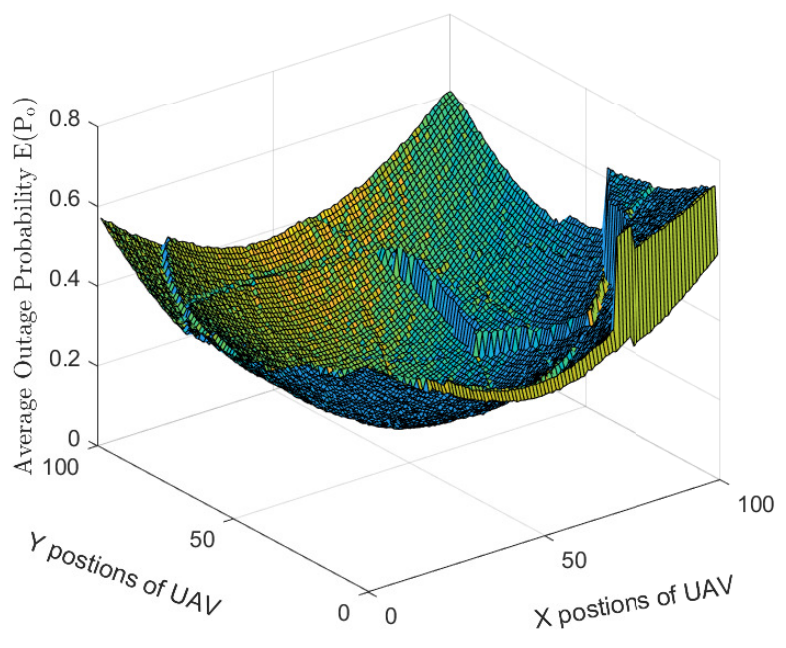

Fig. 2. Move UAV in $x-y$ coordinates versus average outage probability.

with the UAV and MCC on a minimum distance criterion. All the traffic of the $\mathrm{CHs}$ is routed through their associated source (MCC or UAV). Hence, without the presence of any $\mathrm{CN}$, the optimization problem is to find a location for the UAV $\psi(x, y, z)$ which minimizes the outage probability in the network i.e.,

$$
\begin{aligned}
& \min _{\psi(x, y, z)} \mathrm{E}\left(P_{o}\right) \\
& \text { subject to: } 0 \leq(x, y) \leq(X, Y) \\
& z=H_{\text {uav }}
\end{aligned}
$$

The above constraints indicate a scenario in which the OSA devices and MCC are located in 2D and UAV is located in 3D respectively. The height $\left(H_{\text {uav }}\right)$ cannot affect the placement of UAV and is treated as a constant term. Fig. 2 shows the average outage probability at all the positions of UAV $\mathrm{x}-\mathrm{y}$ plane (altitude of the UAV is fixed) in the disaster scenario when $(X, Y)=(100,100)$. The minimal average outage probability is observed between the UAV coordinates $(55,55)$ to $(60,60)$.

It is often observed in the disaster scenario, that some nodes carry more critical data with respect to other nodes or they may be strategically placed in a disaster scenario or they may be the nodes with less energy. Without going into the functionality which makes an OSA critical, we consider the possibility of multiple CNs in a network. In order to provide coverage to these $\mathrm{CNs}$, the UAV placement must consider the outage suffered by the CNs. The percentage of CNs among OSA devices is defined by $\gamma_{c n}$. Since the MCC has no power and energy constraint, therefore, it is possible to increase power from MCC to increase the coverage area and reduce the outage. In some cases such as terrorist attacks, it is not possible for the MCC to increase power as it is not in close proximity to the disaster region. The UAV has power constraints and this is compensated by changing the position of the UAV.
TABLE I

SiMULATION PARAMETER.

\begin{tabular}{|l|l|}
\hline Parameters & Values \\
\hline Area & $100 \mathrm{~m} \times 100 \mathrm{~m}$ \\
\hline$N$ & 100 \\
\hline$\gamma_{m c c}$ & $50-70 \mathrm{~m}$ \\
\hline$\gamma_{c n}$ & $10-40 \%$ \\
\hline $\max .\left(P_{t x}\right)$ & $0.2 \mathrm{Watts}$ \\
\hline$n$ & 3.76 \\
\hline Rayleigh Parameter & $E\left[|x|^{2}\right]=1$ \\
\hline$\beta$ & $0-1$ \\
\hline Noise Power & $-90 \mathrm{dBm}$ \\
\hline
\end{tabular}

We gradually increase the coverage area of MCC $\gamma_{m c c}$ by increasing the transmit power of MCC. The CNs $N_{c n}$ (not in the range of $\gamma_{m c c}$ ) are declared as $\mathrm{CHs}$ with no CMs. These CHs communicate directly with the UAV and the UAV position is adjusted considering the CNs.

For the calculation of the $\mathrm{x}, \mathrm{y}$ coordinates $\mathrm{UAV}$ position based on CNs.

$$
\begin{aligned}
x_{c n} & =\frac{x_{c n, 1}+x_{c n, 2}+\ldots x_{c n, n}}{N_{c n}} \\
y_{c n} & =\frac{y_{c n, 1}+y_{c n, 2}+\ldots . y_{c n, n}}{N_{c n}}
\end{aligned}
$$

The modified UAV position is determine based on $\beta$ which is the ratio factor between $N_{c n}$ and non critical CHs.

$$
\begin{aligned}
& x_{u a v}=\beta x_{c n}+(1-\beta) x_{c h} \\
& y_{u a v}=\beta y_{c n}+(1-\beta) y_{c h}
\end{aligned}
$$

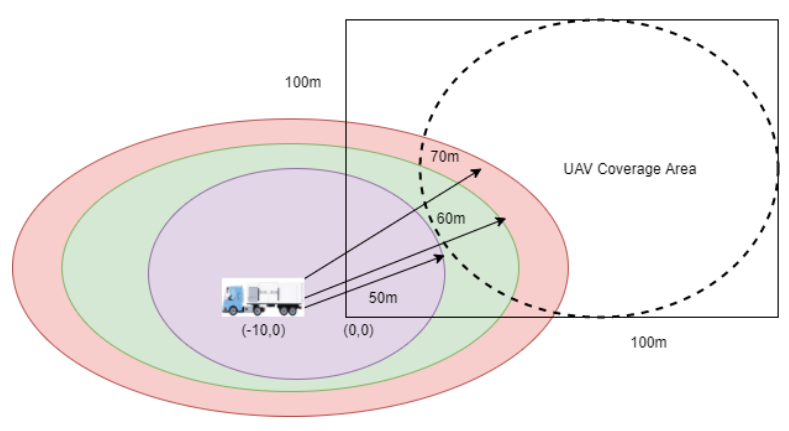

Fig. 3. Simulation Layout.

\section{ANALYTICAL AND SIMULATION RESUlT}

For simulations, we consider an area of $100 \times 100 \mathrm{~m}$ with 100 OSA devices are located uniformly in this area. The OSA devices communicate with the MCC or UAV. We assume the channel between the devices is Rayleigh fading channel, remains constant during one block time, and is i.i.d. We have used MATLAB for the simulations model and the simulation parameters are given in Table I. The MCC is placed at $(-10,0)$ and its coverage range is varied from $50 \mathrm{~m}$ to $70 \mathrm{~m}$ as shown 
in Fig. 3, and the position of UAV is determined based on eqs. 9, 10. Fig. 4 shows the impact of varying transmit power of OSA devices on average outage probability for $\gamma_{c n}=10 \%$.

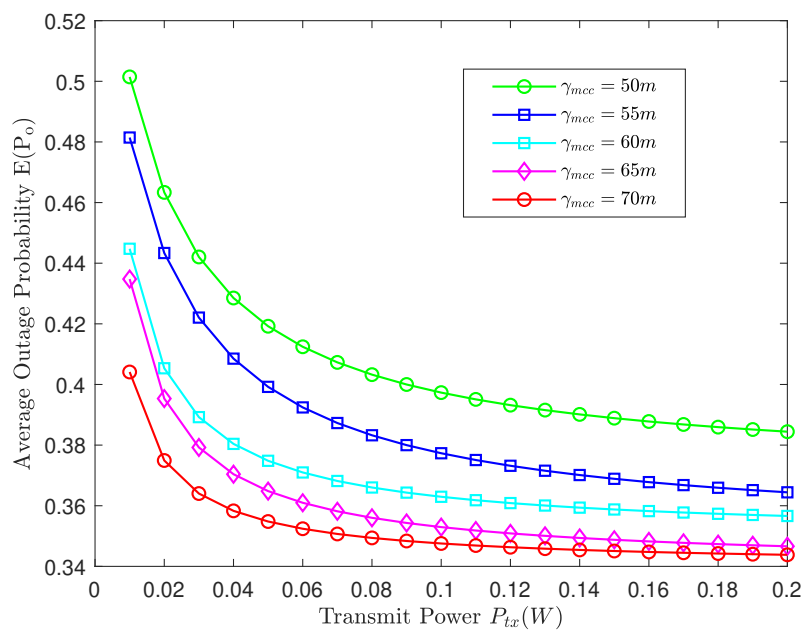

Fig. 4. Average Outage Probability Versus transmit power, $\beta=1, \gamma_{c n}=10 \%$.

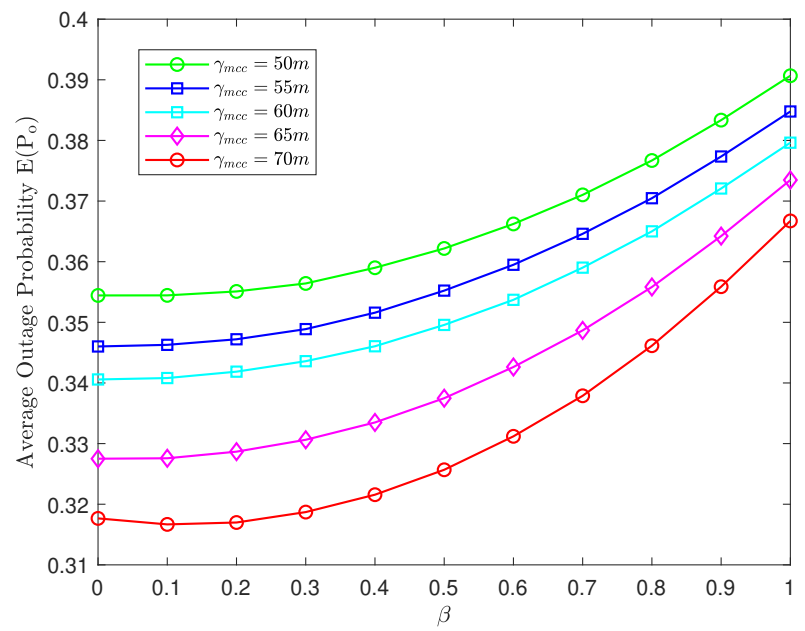

Fig. 5. Effect of $\beta$ on average outage probability, device transmit power $=0.1 \mathrm{~W}, \gamma_{c n}=10 \%$.

This result shows that the average outage probability decreases with the increase in the MCC coverage region. The average outage probability is a minimum for $\gamma_{m c c}$ of $70 \mathrm{~m}$. We cannot further increase the range of $\mathrm{MCC}$ in scenarios like terrorist attacks, hijacking, and etc.

Fig. 5 shows the effect of varying $\beta$ on average outage probability in the case of $\gamma_{c n}=10 \%$. The trend of $\beta$ is the same for all MCC coverage ranges and starts to decrease with the increase in the value of $\beta$. It is interesting to observe that the increase in average outage probability is negligible up to $\beta=0.3$ and starts to increase rapidly beyond 0.3 . In Fig. 6 , we show the effect $\beta$ on average outage probability in the case of $\gamma_{c n}=40 \%$. It is interesting to observe that for

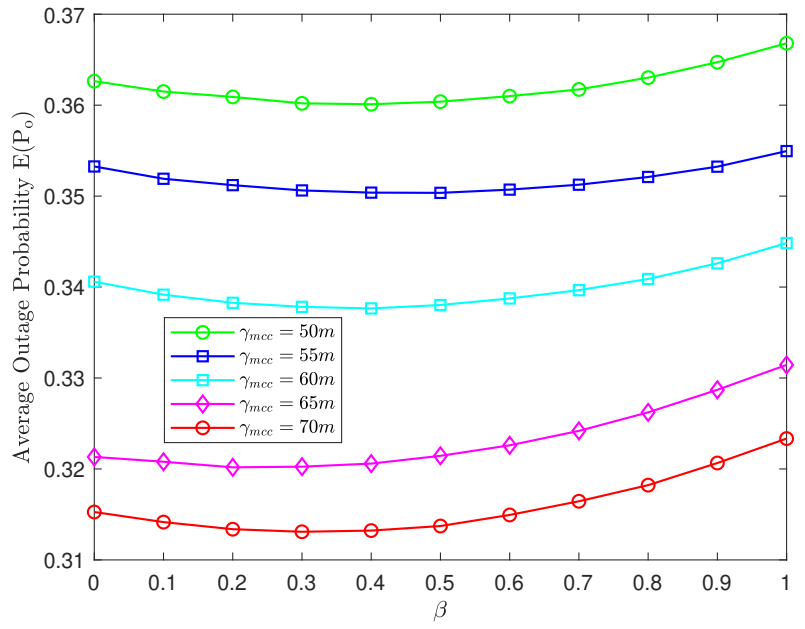

Fig. 6. Effect of $\beta$ on average outage probability, device transmit power of $=0.1 \mathrm{~W}, \gamma_{c n}=40 \%$.

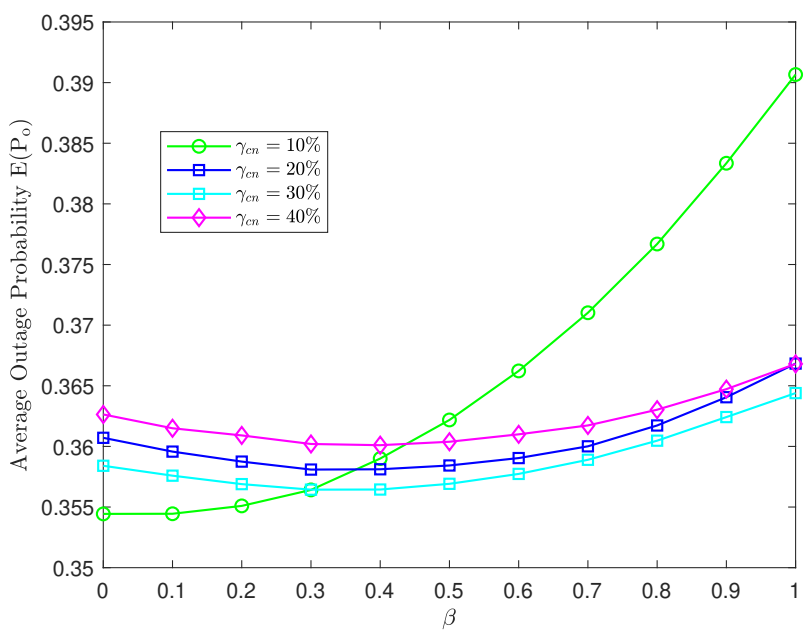

Fig. 7. Effect of $\beta$ on average outage probability, device transmit power $=0.1 \mathrm{~W}, \gamma_{m c c}=50 \mathrm{~m}$.

an increased percentage of $\mathrm{CNs}$, the value of average outage probability decreases for $\beta$ values 0 to 0.4 . The minimum average outage probability is achieved at 0.4 for all MCC coverage ranges. Beyond $\beta=0.4$ the average probability starts to increase gradually.

Fig. 7 depicts the effect of $\beta$ variation on the average outage probability for $\gamma_{m c c}=50 \mathrm{~m}$ and device transmit power of $0.1 \mathrm{~W}$. We see an exceptional trend for the small percentage of CNs i.e. $10 \%$ where average outage probability increases significantly with the value of $\beta$. This trend is not aligned with other percentages of CNs. Next, we observe the same parameters in Fig. 8 for $\gamma_{m c c}=70 \mathrm{~m}$. There is an overall decrease in average outage probability for all $\gamma_{c n}$ as compared to Fig. 7. For $\gamma_{m c c}=70 m$, the UAV associated region shrinks, and the minimal average outage probability is achieved at lower values of $\beta$. This is due to the fact that for smaller 


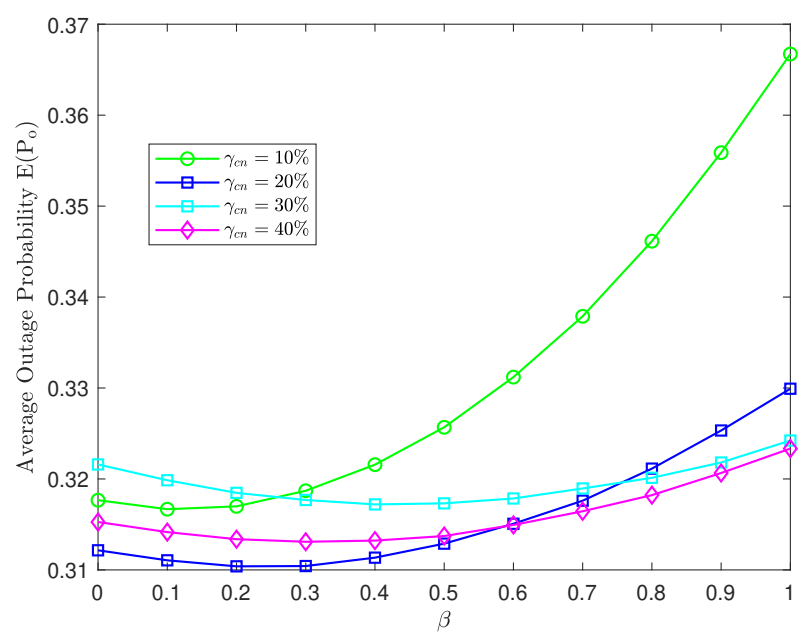

Fig. 8. Effect of $\beta$ on average outage probability, device transmit power $=0.1 \mathrm{~W}, \gamma_{m c c}=70 \mathrm{~m}$.

UAV coverage range the impact of $\mathrm{CNs}$ is not that significant as compared to Fig. 7 .

\section{CONCLUSION}

In this paper, we present a practical UAV assisted model that considers the presence of $\mathrm{CNs}$ in the disaster zone. To manage the trade-off between the connectivity of the clustered nodes and CNs, a weighting coefficient $(\beta)$ is proposed to compute the UAV placement. The results show that when the percentage of CNs is low, the range of MCC has little effect on the average outage, however, the same cannot be said for a higher percentage of CNs. In such a scenario, the value of $\beta$ should be optimized to achieve the minimum probability. The results imply that the UAV under trajectory constraints can use different $\beta$ s to provide coverage to both the clustered and CNs.In the future, we aim to investigate our proposed scheme with multiple UAVs and provision for energy harvesting for CHs and CNs.

\section{ACKNOWLEDGEMENT}

This research has received funding from the NATO-SPS funding grant agreement No. G5482.

\section{REFERENCES}

[1] H. Ritchie, "Natural disasters," Our World in Data, 2014. https://ourworldindata.org/natural-disasters

[2] S. R. Department, "Insured losses caused by man-made catastrophes worldwide 1990-2019 [Online] Available at: ¡https://www.statista.com/statistics/281059/insured-losses-from-manmade-catastrophes-worldwide/i, [Accessed 12-07-2020]."

[3] L. Nasraoui and S. Roy, "Optimal uav positioning for terrestrial users," in 2020 IEEE 91 st Vehicular Technology Conference (VTC2020-Spring), pp. 1-5, IEEE, 2020.

[4] N. Ceccarelli, P. A. Regis, S. Sengupta, and D. Feil-Seifer, "Optimal uav positioning for a temporary network using an iterative genetic algorithm," in 2020 29th Wireless and Optical Communications Conference (WOCC), pp. 1-6, IEEE, 2020.
[5] A. Masood, N. Sharma, M. M. Alam, Y. Le Moullec, D. Scazzoli, L. Reggiani, M. Magarini, and R. Ahmad, "Device-to-device discovery and localization assisted by uavs in pervasive public safety networks," in Proceedings of the ACM MobiHoc workshop on innovative aerial communication solutions for FIrst REsponders network in emergency scenarios, pp. 6-11, 2019.

[6] F. Demiane, S. Sharafeddine, and O. Farhat, "An optimized uav trajectory planning for localization in disaster scenarios," Computer Networks, p. $107378,2020$.

[7] K. Ali, H. Nguyen, Q.-T. Vien, P. Shah, and Z. Chu, "Disaster management using $\mathrm{d} 2 \mathrm{~d}$ communication with power transfer and clustering techniques," IEEE Access, vol. PP, pp. 1-1, 012018.

[8] M. M. Alam, Y. Le Moullec, R. Ahmad, M. Magarini, and L. Reggiani, "A primer on public safety communication in the context of terror attacks: The nato sps "counter-terror" project," in Advanced Technologies for Security Applications, pp. 19-34, Springer, 2020.

[9] L. Özdamar and O. Demir, "A hierarchical clustering and routing procedure for large scale disaster relief logistics planning," Transportation Research Part E: Logistics and Transportation Review, vol. 48, no. 3, pp. 591-602, 2012.

[10] A. M. Hayajneh, S. A. R. Zaidi, D. C. McLernon, M. Di Renzo, and M. Ghogho, "Performance analysis of uav enabled disaster recovery networks: A stochastic geometric framework based on cluster processes," IEEE Access, vol. 6, pp. 26215-26230, 2018.

[11] R. Lalsangi, P. J. Mathew, P. G. Deivapalan, and S. Rao, "Techniques for san storage cluster synchronous disaster recovery," June 232020. US Patent 10,693,955.

[12] Y. Wu, A. Pal, J. Wang, and K. Kant, "Incremental spatial clustering for spatial big crowd data in evolving disaster scenario," in 2019 16th IEEE Annual Consumer Communications \& Networking Conference (CCNC), pp. 1-8, IEEE, 2019.

[13] M. Y. Arafat and S. Moh, "Localization and clustering based on swarm intelligence in uav networks for emergency communications," IEEE Internet of Things Journal, vol. 6, no. 5, pp. 8958-8976, 2019.

[14] A. Masood, D. Scazzoli, N. Sharma, Y. L. Moullec, R. Ahmad, L. Reggiani, M. Magarini, and M. M. Alam, "Surveying pervasive public safety communication technologies in the context of terrorist attacks," Physical Communication, vol. 41, p. 101109, 2020.

[15] S. Chandrasekharan, S. Kandeepan, R. J. Evans, A. Munari, R. Hermenier, M.-A. Marchitti, and K. Gomez, "Clustering approach for aerial base-station access with terrestrial cooperation," in 2013 IEEE Globecom Workshops (GC Wkshps), pp. 1397-1402, IEEE, 2013.

[16] H. I. Minhas, R. Ahmad, W. Ahmed, M. M. Alam, and M. Magarani, "On the impact of clustering for energy critical public safety networks," in 2019 International Symposium on Recent Advances in Electrical Engineering (RAEE), vol. 4, pp. 1-5, IEEE, 2019.

[17] G. Garcia, A. C. K. Vendramin, H. Del Monego, and A. Munaretto, "Lopofly: Location and positioning optimization for flying networks," JOURNAL OF INTELLIGENT \& ROBOTIC SYSTEMS, 2020.

[18] P. V. Klaine, J. P. Nadas, R. D. Souza, and M. A. Imran, "Distributed drone base station positioning for emergency cellular networks using reinforcement learning," Cognitive computation, vol. 10, no. 5, pp. 790804, 2018.

[19] S. Abdellatif, O. Tibermacine, W. Bechkit, and A. Bachir, "Efficient distributed $\mathrm{d} 2 \mathrm{~d}$ prose-based service discovery and querying in disaster situations," in Advanced Information Networking and Applications (L. Barolli, F. Amato, F. Moscato, T. Enokido, and M. Takizawa, eds.), (Cham), pp. 910-921, Springer International Publishing, 2020.

[20] T. M. Kodinariya and P. R. Makwana, "Review on determining number of cluster in k-means clustering," International Journal, vol. 1, no. 6, pp. 90-95, 2013.

[21] L. Yang, J. Chen, M. O. Hasna, and H.-C. Yang, "Outage performance of uav-assisted relaying systems with rf energy harvesting," IEEE Communications Letters, vol. 22, no. 12, pp. 2471-2474, 2018. 